\title{
Inter-individual methylation variability in differentially methylated regions between maternal whole blood and first trimester CVS
}

Marios loannides ${ }^{1,2}$, Elisavet A Papageorgiou ${ }^{1,3}$, Anna Keravnou ${ }^{1}$, Evdokia Tsaliki ${ }^{3}$, Christiana Spyrou ${ }^{3}$, Michael Hadjidaniel ${ }^{1}$, Carolina Sismani ${ }^{1}$, George Koumbaris ${ }^{1,3}$ and Philippos C Patsalis ${ }^{1 *}$

\begin{abstract}
Background: DNA methylation is the most studied form of epigenetic regulation, a process by which chromatin composition and transcription factor binding is altered to influence tissue specific gene expression and differentiation. Such tissue specific methylation patterns are investigated as biomarkers for cancer and cell-free fetal DNA using various methodologies.

Results: We have utilized methylation DNA immunoprecipitation (MeDIP) and real-time quantitative PCR to investigate the inter-individual methylation variability of differentially methylated regions (DMRs) on chromosomes 18 and 21. We have characterized 15 newly selected and seven previously validated DMRs in 50, $1^{\text {st }}$ trimester Chorionic villus samplings (CVS) and 50 female non-pregnant peripheral blood (WBF) samples. qPCR results from MeDIP and genomic DNA (Input) assays were used to calculate fold enrichment values for each DMR. For all regions tested, enrichment was higher in CVS than in WBF samples with mean enrichments ranging from 0.22 to 6.4 and 0.017 to 1 respectively. Despite inter-individual variability, mean enrichment values for CVS were significantly different than those for WBF in all DMRs tested $(p<0.01)$. This observation is reinforced by the absence of overlap in CVS and WBF enrichment value distributions for 15 of 22 DMRs.

Conclusions: Our work provides an expansion in the biomarker panel available for non-invasive prenatal diagnosis (NIPD) using the MeDIP-qPCR methology for Down syndrome and can eventually provide the starting point towards the development for assays towards the detection of Edwards syndrome. Furthermore, our data indicate that inter-experimental and inter-individual variation in methylation is apparent, yet the difference in methylation status across tissues is large enough to allow for robust tissue specific methylation identification.
\end{abstract}

Keywords: Non-invasive prenatal diagnosis, Inter-individual variability, Differentially methylated regions, MeDIP

\section{Background}

In vertebrates DNA methylation is a conserved epigenetic modification by which DNA methyltransferases add a methyl group to carbon 5 of cytosine residues present in CpG dinucleotides. This modification is the most studied form of epigenetic regulation and has been strongly associated with chromosomal stability and imprinting control [1]. Furthermore, this epigenetic process also regulates chromatin composition and transcription factor binding to directly influence transcriptional activity $[2,3]$.

\footnotetext{
* Correspondence: patsalis@cing.ac.cy

'The Cyprus Institute of Neurology and Genetics, Nicosia, Cyprus Full list of author information is available at the end of the article
}

DNA methylation occurs primarily in $\mathrm{CpG}$ islands (GGIs) and shores both in coding and non-coding regions of the genome, with gene regulatory regions such as promoters and first exons being a frequent methylation target [4]. Due to this integral relationship with gene expression regulation, DNA methylation patterns are very closely associated with developmental processes and differentiation. Consequently, DNA methylation directly modulates phenotype, and distinct methylation patterns have been associated with tissue specificity and a variety of disease states ranging from cancer to neurological disorders [5,6]. These tissue specific differentially methylated regions (tDMRs) are currently under investigation for 
their utility as biomarkers for disease progression and prognosis, particularly in the field of cancer research, disease detection and response to treatment [7].

The discovery of cell free fetal DNA (cffDNA) in the maternal circulation has greatly facilitated the development of non-invasive prenatal diagnosis (NIPD) [8]. The direct correlation between phenotype and DNA methylation patterns has allowed the use of DMRs as possible biomarkers in prenatal diagnosis. Several groups have utilized the methylation differences between placentaderived cffDNA and maternal DNA in order to identify highly specific fetal DMR biomarkers for non-invasive prenatal diagnosis of aneuploidies. Previous studies employed a variety of methods including sodium bisulfite conversion and methylation sensitive restriction digestion, but yielded a relatively small number of fetal specific DMRs including the SERPINB5, RASSF1A and U-PDE9A genes [9-11].

In 2009, Papageorgiou et al. [12] applied methylation DNA immunoprecipitation (MeDIP) coupled with high resolution tiling oligonucleotide array (Chip) analysis to identify DMRs between Chorionic villus sampling (CVS) and female peripheral blood DNA (WBF). They were able to identify thousands of DMRs on chromosomes 13, 18, $21, \mathrm{X}$, and $\mathrm{Y}$ including methylation sensitive restriction sites, CGIs and promoter regions. This MeDIP-Chip approach was the trigger for investigating the utility of MeDIP followed by real-time qPCR (MeDIP-qPCR) for the non-invasive prenatal diagnosis of trisomy 21 , yielding $100 \%$ sensitivity and specificity [13]. This novel NIPD method was validated by a second study of 175 cases again yielding high sensitivity and specificity [14].

The current study utilizes the MeDIP-qPCR methodology to expand our range of fetal specific DMR biomarkers by selecting and screening 15 additional DMRs on chromosomes 21 and 18. Special emphasis is given on investigating the methylation variability in different samples from these newly selected and previously reported DMRs [12-14] by screening them in a set of $50,1^{\text {st }}$ trimester CVS and 50 WBF. Overall, this work confirms the distinctively different methylation status of these regions in CVS and WBF.

\section{Results}

Using the above criteria we identified a set of 40 candidate DMRs between CVS and WBF from the microarray data [12]. This set was subsequently screened in a cohort of six CVS and six WBF to calculate the enrichment values for each DMR (Additional file 1). Based on this initial screening we were able to select the 15 regions with the highest CVS enrichment for further validation/ characterization, using seven previously validated DMRs by Papageorgiou et al. [13] and Tsaliki et al. [14], as a comparison standard.
This DMR validation study was conducted on a set of 50 CVS and 50 WBF samples using the MeDIP-qPCR methodology (Table 1), the efficiency of which was monitored using one hypermethylated (HYPER) and one hypomethylated (HYPO) control regions. The HYPER is a region that showed hypermethylation for both CVS and WBF, while the HYPO is a region that showed hypomethylation for the two tissues [12]. Enrichment values for HYPO were low in WBF and CVS samples while the HYPER control region showed enrichment for CVS and WBF with mean enrichment values of 3.12 and 3.22 respectively, indicating that the MeDIP procedure was highly specific for the methylated regions. Moreover, the previously validated DMRs performed as previously described [12], exhibiting distinctively different enrichment between CVS and WBF.

All tested DMRs showed a significant enrichment $(\mathrm{p}<$ 0.01) in CVS compared to those of WBF (Table 1). We compared the performance of the 15 newly selected DMRs with the previously validated set and we were able to determine that 11 of 15 DMRs showed enrichment values higher than the lowest of the previously validated DMRs, ranging from 1.9 to 6.4. Additional comparison of the two DMR sets also illustrated that for 11 of these 15 regions the difference of means (mean enrichment CVS mean enrichment WBF) was again higher than the respective values of the validated DMRs (ranging from 1.6 to 6.4) (Table 1). Our analysis also shows that the enrichment distributions for CVS and WBF have no overlap for these 11 DMRs (Figure 1).

To better investigate tissue specificity (CVS-WBF) in the 15 newly selected DMRs in relation to the previously validated DMRs, we also constructed a heat map and hierarchical clustering of the 50 CVS and 50 WBF samples based on the obtained enrichment values (Figure 2). This analysis shows a clear differentiation between the two tissue types based on the obtained enrichment values. Furthermore, DMR clustering analysis showed that there was no distinct clustering separation between the newly selected and the previously validated DMRs.

\section{Discussion}

Our study aimed to validate and characterize a set of differentially methylated regions between CVS and WBF, obtained from MeDIP-Chip data [12]. The methylation characteristics of the 15 candidate DMRs, located on chromosomes 18 and 21, were ascertained in 50 CVS and 50 WBF using the MeDIP-qPCR methodology. To our knowledge this is the first MeDIP based biomarker screening study utilizing such a large sample set. None of the selected DMRs were located on CGIs, but within intergenic or intragenic regions. Such DMR distribution in noncoding intergenic and intragenic sequences is in agreement with data from a large scale investigation of tissue 
Table 1 Ranking of DMRs tested according to the difference between mean enrichment values for each DMRs

\begin{tabular}{|c|c|c|c|c|c|c|c|c|}
\hline Marker & Mean WBF & Mean CVS & Mean difference & SD WBF & SD CVS & U pval & Coefficient of variation WBF & Coefficient of variation CVS \\
\hline El-4 & 0.017 & 6.384 & 6.367 & 0.022 & 2.143 & $1.53 \mathrm{E}-17$ & 1.294 & 0.336 \\
\hline Ell-1 & 0.065 & 5.319 & 5.254 & 0.071 & 1.937 & $7.06 \mathrm{E}-18$ & 1.092 & 0.364 \\
\hline $\mathrm{H} 2$ & 0.135 & 4.068 & 3.933 & 0.093 & 1.252 & $7.06 \mathrm{E}-18$ & 0.689 & 0.308 \\
\hline $\mathrm{El}-2$ & 0.064 & 3.894 & 3.83 & 0.2 & 1.338 & $7.50 \mathrm{E}-18$ & 3.125 & 0.344 \\
\hline $\mathrm{El}-3$ & 0.116 & 3.905 & 3.789 & 0.312 & 1.556 & $1.96 \mathrm{E}-17$ & 2.690 & 0.398 \\
\hline B3 & 0.126 & 3.86 & 3.734 & 0.1 & 1.268 & $2.29 \mathrm{E}-17$ & 0.794 & 0.328 \\
\hline M27 & 0.532 & 4.113 & 3.581 & 0.2 & 1.386 & 7.06E-18 & 0.376 & 0.337 \\
\hline D2 & 0.317 & 3.364 & 3.047 & 0.179 & 1.301 & 7.06E-18 & 0.565 & 0.387 \\
\hline M28 & 0.189 & 2.777 & 2.588 & 0.125 & 0.919 & 7.06E-18 & 0.661 & 0.331 \\
\hline M1E & 0.149 & 2.636 & 2.487 & 0.097 & 0.742 & $3.44 \mathrm{E}-17$ & 0.651 & 0.281 \\
\hline Id1 & 0.398 & 2.682 & 2.284 & 0.155 & 0.932 & $1.04 \mathrm{E}-17$ & 0.389 & 0.348 \\
\hline A5 & 0.337 & 2.505 & 2.168 & 0.159 & 0.986 & 7.06E-18 & 0.472 & 0.394 \\
\hline $\mathrm{C} 5$ & 0.18 & 2.321 & 2.141 & 0.106 & 0.84 & 7.06E-18 & 0.589 & 0.362 \\
\hline $\mathrm{C} 1$ & 0.106 & 2.229 & 2.123 & 0.083 & 0.635 & 7.06E-18 & 0.783 & 0.285 \\
\hline All-2 & 0.065 & 2.003 & 1.938 & 0.084 & 0.933 & $1.23 \mathrm{E}-16$ & 1.292 & 0.466 \\
\hline On2 & 0.281 & 1.993 & 1.712 & 0.138 & 0.552 & $7.06 \mathrm{E}-18$ & 0.491 & 0.277 \\
\hline $\mathrm{Nn} 2$ & 0.245 & 1.924 & 1.679 & 0.107 & 0.78 & 7.07E-18 & 0.437 & 0.405 \\
\hline$J 2$ & 0.116 & 1.707 & 1.591 & 0.079 & 0.519 & 7.06E-18 & 0.681 & 0.304 \\
\hline Fd1 & 0.135 & 1.676 & 1.541 & 0.101 & 0.513 & $7.06 \mathrm{E}-18$ & 0.748 & 0.306 \\
\hline M25 & 1.038 & 1.822 & 0.784 & 0.452 & 0.655 & $1.88 \mathrm{E}-09$ & 0.435 & 0.359 \\
\hline M20 & 0.42 & 0.796 & 0.376 & 0.186 & 0.303 & $2.37 \mathrm{E}-10$ & 0.443 & 0.381 \\
\hline M18 & 0.097 & 0.22 & 0.123 & 0.07 & 0.151 & 6.36E-08 & 0.722 & 0.686 \\
\hline HYPER & 3.124 & 3.226 & 0.102 & 0.68 & 0.982 & 0.951 & 0.218 & 0.304 \\
\hline HYPO & 0.469 & 0.508 & 0.039 & 0.945 & 0.948 & 0.119 & 2.015 & 1.866 \\
\hline
\end{tabular}

Despite the statistical significance of all enrichment values $(p<0.01)$, the four markers $(M 25, M 20, M 18, F d 1)$ that showed the lowest difference were not selected as potential DMRs.

specific methylation profiles by Rakyan et al. [15], who reported differential methylation of intergenic and $\mathrm{CpG}$ poor promoter regions in addition to CGIs.

Based on our results, all 15 DMRs showed differential enrichment between the two tissues and 11 out of these 15 were strongly and consistently hypermethylated in CVS samples. The ability of these DMRs to distinguish between CVS and WBF was equivalent to that of the seven previously validated DMRs used as performance standards. In fact, the tissue discriminating performance of the DMRs tested here, shows close similarities with the previously validated DMRs as it is illustrated by our heat map distribution and the unsupervised clustering patterns obtained.

DMR enrichment values showed variability among the different samples. This is likely caused by combination of both inter-experimental technical variability and inter-individual methylation variability. The presence of variability in MeDIP based assays has previously been described by Butcher et al. [16]. In addition, the issue of inter-individual DNA methylation variability has been the focus of several studies [17-19]. This high interindividual variability has been attributed to a variety of factors including environmental conditions, diet, age and psychosocial factors [20-23]. Furthermore, it has been documented that regions with low $\mathrm{CpG}$ density, as the DMRs under investigation, show higher inter-individual variability as compared to regions with high CpG density, such as CGIs [24].

Others have also shown that methylation variability can coincide with tissue specific DMRs without obscuring the tissue discriminating properties of those DMRs [17]. It is therefore of no surprise that despite the DNA methylation variability in our study, the newly validated set of 11 DMRs clearly distinguishes between CVS and WBF tissues.

Our work here substantially increases the number of confirmed chromosome 21 fetal specific DMRs, and therefore provides a significant expansion in the biomarker panel available for MeDIP-qPCR-based NIPD of Down syndrome. Such an expansion is predicted to further improve the robustness of the methodology and bolster its diagnostic classification power. It is also very important to 


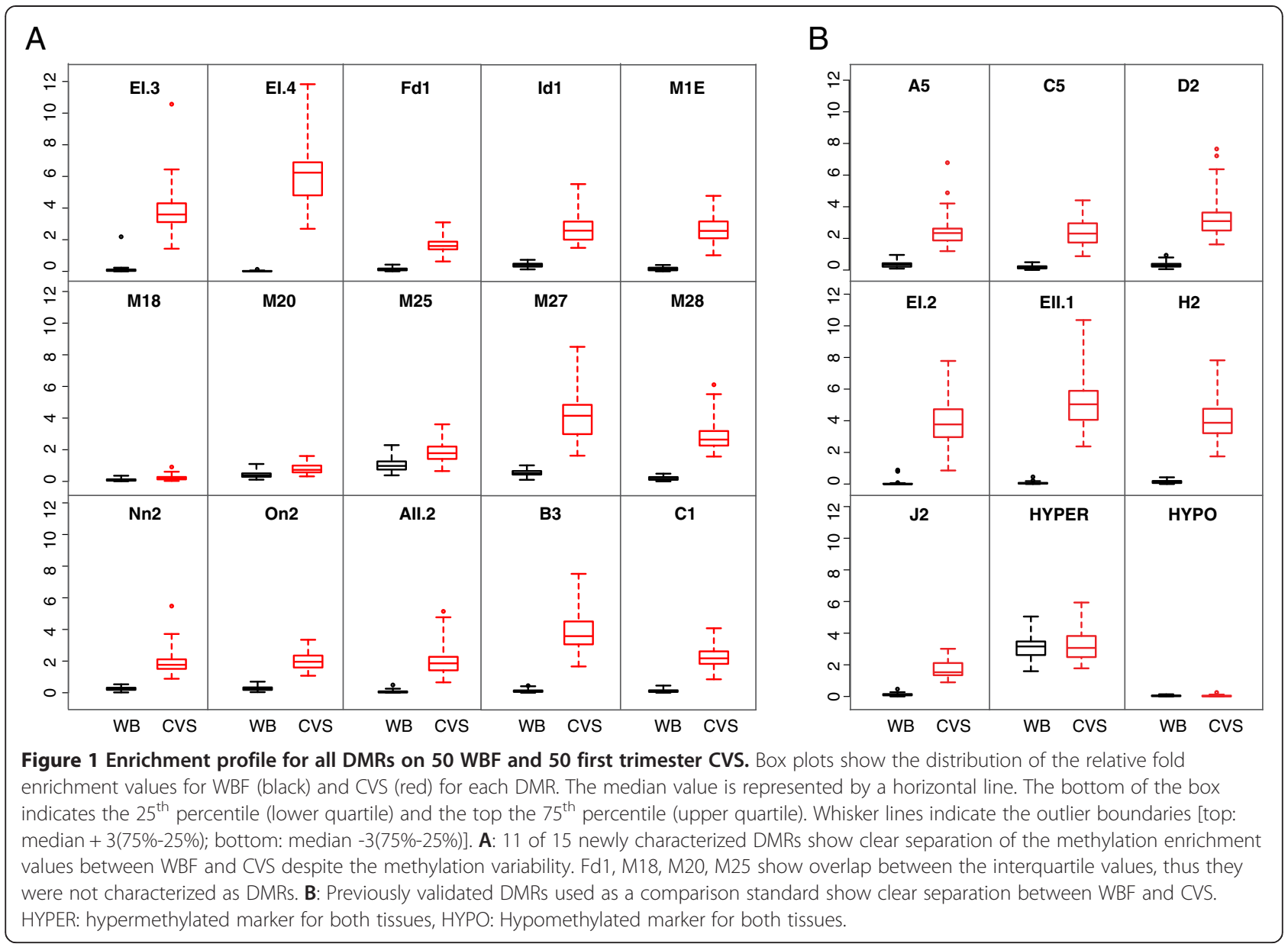

note that our current study is the first to validate chromosome 18 fetal specific DMRs in a relatively large sample set. This small panel of chromosome 18 DMRs can potentially provide a very valuable testing platform on which future NIPD assays for Edwards syndrome will be developed.

\section{Conclusions}

NIPD has gained a lot of interest the last few years. Utilizing the methylation differences between fetal and maternal DNA, several groups have managed to identify biomarkers using different approaches. This study aimed to characterize and validate fetal specific methylated regions using the MeDIP-qPCR methodology. We were able to show that the selected regions had distinct methylation patterns between fetal and maternal tissue, despite interindividual and inter-experimental variability. In addition, we have expanded the panel of the existing DMRs on chromosome 21 and have characterized a new set of markers on chromosome 18 which can provide the starting point towards the development for assays towards the detection of Edwards syndrome.

\section{Methods}

\section{Human Samples and DNA preparation}

WBF samples were obtained anonymously from 50 normal non-pregnant females $20-40$ years of age. Fifty, $1^{\text {st }}$ trimester CVS were obtained from the Department of Cytogenetic and Genomics at the Cyprus Institute of Neurology and Genetics (Nicosia, Cyprus). Protocols used for collecting samples for our study were approved by the appropriate Bioethics Committees, and informed consent was obtained from all participants. WBF and CVS samples were used to extract DNA using the QIAamp DNA blood midi kit or the QIAmp DNA mini kit according to the manufacturer's instructions (QIAGEN, Hilden, Germany). All CVS underwent karyotyping and Quantitative-Fluorescent PCR (QF-PCR) analysis in order to confirm their normal status.

\section{Ligation-mediated PCR (LM-PCR) and MeDIP assay}

LM-PCR and MeDIP assays were conducted as described previously [12]. Briefly, $2.5 \mu \mathrm{g}$ of genomic DNA were sonicated using the Bioruptor Twin sonication system (UCD-400, Diagenode, Liege, Belgium) into fragments, 300-1000 bp in size. Fragment size was verified 


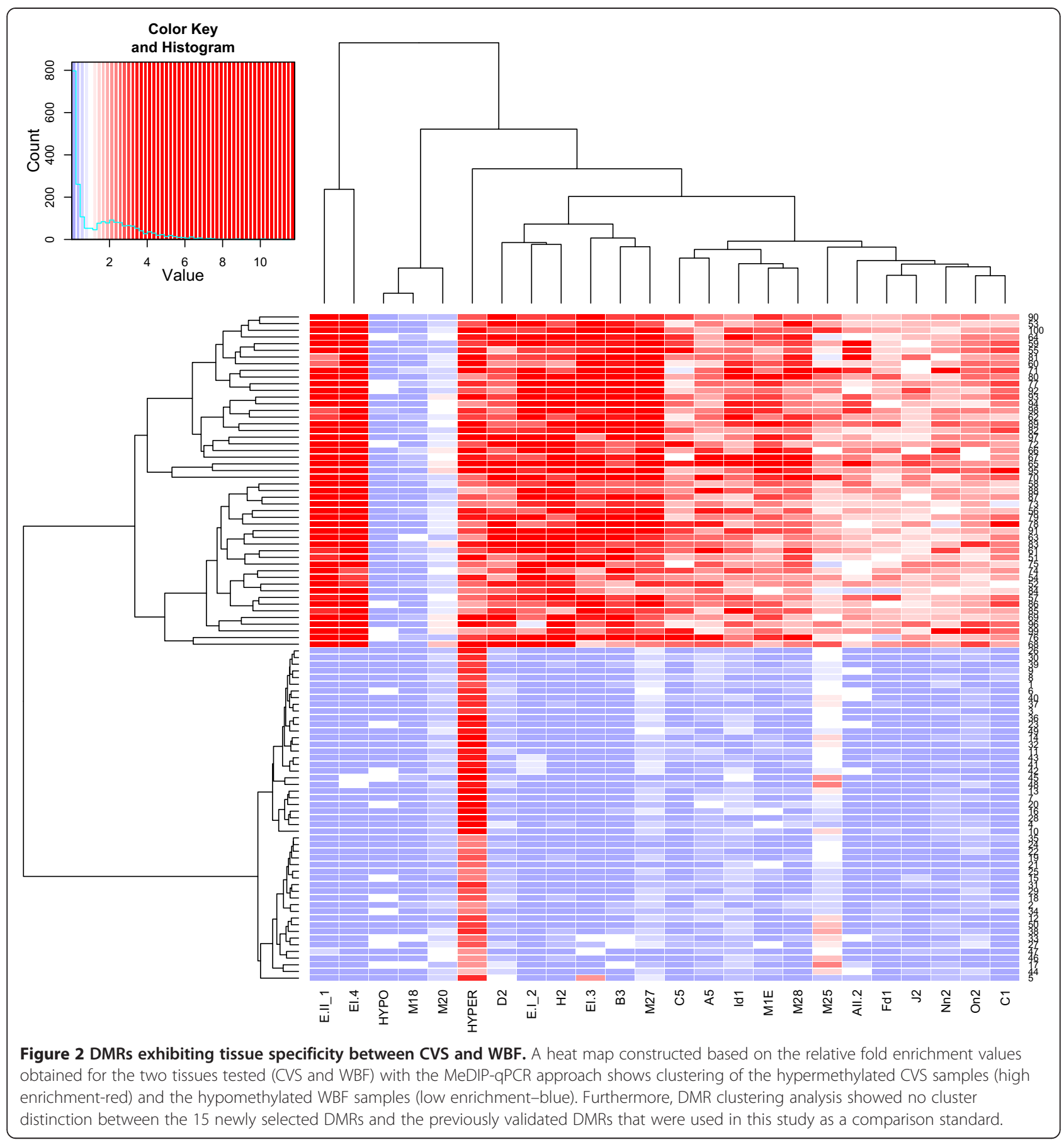

using agarose gel electrophoresis. The fragments were blunt-ended using HPLC water, 1X NEB buffer 2 (New England BioLabs, Ipswich, UK), 10X bovine serum albumin (New England BioLabs) $100 \mathrm{mmol} / \mathrm{L}$ dNTP mix (GE Healthcare, Little Chalfont, UK) and T4 DNA polymerase ( $3 \mathrm{U} / \mu \mathrm{l}$; New England BioLabs). Fragments were purified using the QiAquick PCR purification kit (Qiagen) and linkers were then ligated onto the blunt ends by overnight incubation at $16^{\circ} \mathrm{C}$ with T4 DNA ligase (New England) and T4 DNA ligase buffer (New England). Overhangs were subsequently filled in by incubating at $72^{\circ} \mathrm{C}$ for 10 minutes with $100 \mathrm{mmol} / \mathrm{L}$ dNTP mix (GE Healthcare), 1X PCR gold buffer (Roche, Mannheim, Germany), $1.5 \mathrm{mmol} / \mathrm{L} \mathrm{MgCl}_{2}$ (Roche) HPLC water and AmpliTaq DNA polymerase (Applied Biosystems, Branchburg, New Jersey, USA). $50 \mathrm{ng}$ of ligated DNA was removed and 
kept as input DNA. The remaining ligated DNA (800$1200 \mathrm{ng}$ ) was subjected to MeDIP using $3 \mu \mathrm{g}$ mouse anti5 'methylCytosine $(\mathrm{a}-5 \mathrm{mC})$ antibody (Eurogentec Saraing, Belgium). Hypermethylated DNA bound to a-mC antibodies was magnetically captured using Dynabeads ${ }^{\circ}$ M-280 Sheep Anti-Mouse IgG magnetic beads (Life technologies, Carlsbad, California, USA) and subsequently released using proteinase $\mathrm{K}$ (Roche). LM-PCR used $12 \mathrm{ng}$ of each input and MeDIP DNA as described earlier [12].

\section{DMR selection}

Candidate DMRs on chromosomes 18 and 21 were selected from a set of potential differentially methylated regions previously described [12] according to the following three criteria: a) the region included at least three consecutive microarray probes, b) array results showed consistent DNA hypermethylation in first and third trimester placentas and hypomethylation in WBF samples, c) the region did not include segmental duplications and copy number variable regions based on the Database for Genomic Variants (DGV) [25]. The regions considered for this paper are shown in Table 2.

\section{Real-Time quantitative PCR (qPCR)}

Primer design, optimal primer concentration experiments, and efficiency (e) of each qPCR reaction were performed as previously described [12] with the following modifications. Each qPCR reaction was performed on $8 \mathrm{ng}$ of template DNA using SYBR Green PCR mastermix (Eurogentec) in a final reaction volume of $10 \mu \mathrm{l}$, using a BIORAD CFX 384 Real time system (BIORAD, Hercules, California). Each MeDIP, or Input template DNA was used to prepare three replicate qPCR reactions that were used for calculating the average $\mathrm{Ct}$ value for each template. Primer3 software [26] was used to design the qPCR primer sets that were synthesized by Sigma-Aldrich (Munich, Germany). Primer sets utilized for this body of work are listed in Table 3.

\section{Statistical calculations}

MeDIP enrichment values of the CVS and WBF samples were calculated for each region using the following equation:

Enrichment $=\mathbf{e}^{\Delta \mathrm{Ct}}$ where e corresponds to the efficiency obtained in each real-time PCR reaction e $=10^{(-1 /}$ slope of STD curve) ) and $\Delta \mathrm{Ct}$ indicates the cycle difference between input DNA and MeDIP DNA $\left[\mathrm{Ct}_{(\mathrm{IN})}-\mathrm{Ct}_{(\mathrm{IP})}\right]$.

Table 2 Characteristics of the regions tested

\begin{tabular}{|c|c|c|c|}
\hline Chromosomal region & Position (hg18) & Location type & Gene involved \\
\hline $\mathrm{Nn}$ & chr21:31426757-31427146 & Intragenic & TIAM1 \\
\hline $\mathrm{H}$ & chr21:32268787-32269137 & Intragenic & HUNK \\
\hline C & chr21:33320530-33320815 & CpG Island & OLIG2 \\
\hline On & chr21:34492714-34493203 & Intergenic & \\
\hline J & chr21:37841231-37841506 & Intergenic & \\
\hline A & chr21:39279691-39279971 & Intergenic & \\
\hline $\mathrm{Fd}$ & chr21:42005961-42006216 & Intragenic & C21orf129 \\
\hline M27 & chr21:42178808-42179008 & Intragenic & $\mathrm{C} 2 \mathrm{CD} 2$ \\
\hline D & chr21:42189235-42189849 & LINE-L1 & $\mathrm{C} 2 \mathrm{CD} 2$ \\
\hline EI & chr21:42355366-42355908 & Intergenic & \\
\hline Ell & chr21:42357141-42357401 & Intergenic & \\
\hline ld & chr21:42753677-42754026 & Intergenic & \\
\hline $\mathrm{M} 1 \mathrm{E}$ & chr21:44953640-44953854 & Intragenic & TSPEAR \\
\hline$M 28$ & chr21:45171015-45171225 & Intragenic & ITGB2 \\
\hline $\mathrm{Al}$ & chr18:55086179-55086755 & Intragenic & RAX NM-013435 \\
\hline All & chr18:55090284-55090605 & Intragenic & RAX NM-013435 \\
\hline B & chr18:44165984-44166275 & Intergenic & \\
\hline C & chr18:58955844-58956604 & Intragenic & BCL2NM-000633 \\
\hline M18 & chr21:15331818-15331945 & Intragenic & NR1P1 \\
\hline M20 & chr21:15178413-15178497 & Intergenic & \\
\hline M25 & chr21:37692864-37692974 & Intergenic & DYRK1A \\
\hline
\end{tabular}

Regions in bold indicate previously validated regions $[13,14]$. 
Table 3 Primer sequences on DMRs tested

\begin{tabular}{|c|c|c|c|}
\hline Primer & Forward & Reverse & Position \\
\hline CHR21(M27) & ATACGTGTCCTGCCTTCCAC & GCTITGAGCAGAGAGGGAAA & $42178812-42178948$ \\
\hline CHR21(M28) & CCCAGAAATTCCATTTGCAG & GAAAGGCTCAACCAACCAAC & $45171107-45171192$ \\
\hline CHR21(M1E) & TCGCACTGAGGCTTCCTACT & AAGTTGTGGGCTGGGATTT & $44953674-44953772$ \\
\hline CHR21(Nn2) & ACCATTGTGGATCACAGCAG & GCTCCGAGGATTAGGGAAAG & $31427008-31427139$ \\
\hline CHR21(On2) & СTCCTGACCCACTCCCAATA & GGAAACTCAGGGTCAAACGA & $34492982-34493090$ \\
\hline CHR21(Fd1) & ATGTTGCCTGGGATATGCTT & AACTGGCTGCGTGAGGATA & $42006045-42006153$ \\
\hline CHR21(El-3) & GCCTTGGGACAAAAATGACA & TGGGCACAGCCCTAACTAAC & $42355352-42355484$ \\
\hline CHR21(El-4) & GGCCAGGTTGTTTCAGATTG & TTCCGGCAGAGTTTATTTGG & $42355802-42355908$ \\
\hline CHR21(Id1) & ACCGTATCATTTCCCCAGGT & TGACCACATTTCCACCACAG & $42753720-42753866$ \\
\hline CHR21(A5) & GCTGGACCAGAAAGTGTTGAG & GTGTGCTGCTTTGCAATGTG & $39279856-39280004$ \\
\hline CHR21(C5) & CTGTTGCATGAGAGCAGAGG & CGTCCCCCTCGCTACTATCT & $33320735-33320829$ \\
\hline CHR21(D2) & TGCAGGATATTTGGCAAGGT & CTGTGCCGGTAGAAATGGTT & $42189557-42189683$ \\
\hline CHR21(EI-2) & TGAATCAGTTCACCGACAGC & GAAACAACCTGGCCATTCTC & $42355712-42355815$ \\
\hline CHR21(EII-1) & CCGTTATATGGATGCCTTGG & AAACTGTTGGGCTGAACTGC & $42357215-42357341$ \\
\hline CHR21(H2) & ССАСАТСCTGGCCATCTACT & TTCCACAGACAGCAGAGACG & $32268843-32268943$ \\
\hline CHR21(M18) & GATGGATGGCCTITTGGTAA & TATTTGGTTGCCCCTTCCT & 15331818-5331945 \\
\hline CHR21(M20) & CATTAGCGGGTCAGCTAGGA & TGGCAATTACATCTGCCATTA & $15178413-5178497$ \\
\hline CHR21(M25) & TTGTCTGCCCGTATGGAAGT & ATGGTTGTAGGGCTCATTCA & $37692864-37692974$ \\
\hline CHR21(J2) & ATTCTCCACAGGGCAATGAG & TTATGTGGCCTTTCCTCCTG & $37841284-37841411$ \\
\hline CHR18(All2) & TGTGCCTCTCCCTTGAGACT & AAATTGCAGCCAATGCTTCT & $55090427-55090524$ \\
\hline CHR18(B3) & TGTGGTTTCAAACATGCACA & CTGAAAAGGCCACTCTGAGG & $44166131-44166263$ \\
\hline CHR18(C1) & GTGAGAGAGAACGCCAGGAG & TGAGCCAACTCTGGTGTCAG & $58956266-58956391$ \\
\hline HYPER & CAGGAAAGTGAAGGGAGCTG & CAAAACCCAATGGTCAATCC & 19991387-19991465 \\
\hline HYPO & AGGTGCCCAATTCAAGGTA & CTTCCCCACCAGTCTTGAAA & $30214952-30215055$ \\
\hline
\end{tabular}

Regions in bold indicate previously validated regions $[13,14]$.

The mean enrichment values of each DMR were compared between WBF and CVS samples using the MannWhitney $U$ tests [27] and the corresponding p-values were used to decide whether there was significant evidence to claim that the mean enrichments of the two groups were different.

Hierarchical clustering of the DMRs was conducted using an iterative algorithm that joins similar clusters based on the set of dissimilarities of the 100 individuals (calculating the Euclidean distanced between clusters) and re-computing their distances at each stage by the Lance-Williams dissimilarity update formula [28].

\section{Additional file}

Additional file 1: Initial screening on six WBF and six CVS for the selection of new DMRs.

\section{Competing interests}

GK and EAP are currently employed by, and own shares of NIPD Genetics LTD which is developing a new non-invasive prenatal diagnosis for trisomy 21 based on the MeDIP-qPCR methodology. MI, AK, MH and CS declare that they have no competing interests.
At the time that the study was carried out ET and CS were employed by NIPD Genetics LTD. Currently, they have no affiliations with NIPD Genetics LTD and they declare no competing interests.

EAP and PCP have filed a PCT patent application for the MeDIP real time qPCR based NIPD approach (PCT Patent Application No.

PCT/1B2011/000217)

\section{Authors' contributions}

$\mathrm{Ml}$ assisted in the design of the study, collected samples, carried out all the experiments and drafted the manuscript. EAP assisted in the design of the study. AK participated in the Methylation DNA Immunoprecipitation and qPCR assays. ET assisted in the biomarker discovery. CS carried out the statistical analysis. CS assisted in the design of the study. GK and $\mathrm{MH}$ assisted in the design of the study and helped to edit the manuscript. PCP conceived and coordinated the study. All authors read and approved the final manuscript.

\section{Acknowledgements}

The authors would like to thank Andrea Chrysostomou for her assistance in MeDIP and GPCR assays. This research was partially funded by the 7th Framework Programme as part of the ANGELAB project (http://cordis.europa. eu/projects/rcn/105552 en.html, project number 317635).

\section{Author details}

${ }^{1}$ The Cyprus Institute of Neurology and Genetics, Nicosia, Cyprus. ${ }^{2}$ Department of Biological Sciences, University of Cyprus, Nicosia, Cyprus. ${ }^{3}$ NIPD Genetics Ltd, Nicosia, Cyprus. 
Received: 22 September 2014 Accepted: 12 October 2014

Published online: 01 November 2014

\section{References}

1. Bird A: DNA methylation patterns and epigenetic memory. Genes Dev 2002, 16:6-21.

2. Gopalakrishnan S, Van Emburgh BO, Robertson KD: DNA methylation in development and human disease. Mutat Res 2008, 647:30-38.

3. Baylin SB, Jones PA: A decade of exploring the cancer epigenomebiological and translational implications. Nat Rev Cancer 2011, 11:726-734.

4. Laird PW: Principles and challenges of genomewide DNA methylation analysis. Nat Rev Cancer 2010, 11:191-203.

5. You JS, Jones PA: Cancer genetics and epigenetics: two sides of the same coin? Cancer Cell 2012, 22:9-20.

6. Jakovcevski M, Akbarian S: Epigenetic mechanisms in neurological disease. Nat Med 2012, 18(8):1194-1204.

7. Rawson JB, Bapat B: Epigenetic biomarkers in colorectal cancer diagnostics. Expert Rev Mol Diagn 2012, 12:499-509.

8. Lo YM, Corbetta N, Chamberlain PF, Rai V, Sargent IL, Redman CW, Wainscoat JS: Presence of fetal DNA in maternal plasma and serum. Lancet 1997, 350:485-487.

9. Della Ragione F, Mastrovito P, Campanile C, Conti A, Papageorgiou EA, Hulten MA, Patsalis PC, Carter NP, D'Esposito M: Differential DNA methylation as a tool for noninvasive prenatal diagnosis (NIPD) of $X$ chromosome aneuploidies. J Mol Diagn 2010, 12:797-807.

10. Old RW, Crea F, Puszyk W, Hulten MA: Candidate epigenetic biomarkers for non-invasive prenatal diagnosis of Down syndrome. Reprod Biomed Online 2007, 15:227-235.

11. Tong YK, Chiu RW, Akolekar R, Leung TY, Lau TK, Nicolaides KH, Lo YM: Epigenetic-genetic chromosome dosage approach for fetal trisomy 21 detection using an autosomal genetic reference marker. PLoS One 2010, 5:e15244.

12. Papageorgiou EA, Fiegler H, Rakyan V, Beck S, Hulten M, Lamnissou K, Carter $N P$, Patsalis PC: Sites of differential DNA methylation between placenta and peripheral blood: molecular markers for noninvasive prenatal diagnosis of aneuploidies. Am J Pathol 2009, 174:1609-1618.

13. Papageorgiou EA, Karagrigoriou A, Tsaliki E, Velissariou V, Carter NP, Patsalis PC: Fetal-specific DNA methylation ratio permits noninvasive prenatal diagnosis of trisomy 21. Nat Med 2011, 17:510-513.

14. Tsaliki E, Papageorgiou EA, Spyrou C, Koumbaris G, Kypri E, Kyriakou S, Sotiriou C, Touvana E, Keravnou A, Karagrigoriou A, Lamnissou K, Velissariou V, Patsalis PC: MeDIP real-time qPCR of maternal peripheral blood reliably identifies trisomy 21. Prenat Diagn 2012, 32:996-1001.

15. Rakyan VK, Down TA, Thorne NP, Flicek P, Kulesha E, Graf S, Tomazou EM, Backdahl L, Johnson N, Herberth M, Howe KL, Jackson DK, Miretti MM, Fiegler H, Marioni JC, Birney E, Hubbard TJ, Carter NP, Tavare S, Beck S: An integrated resource for genome-wide identification and analysis of human tissue-specific differentially methylated regions (tDMRs). Genome Res 2008, 18(9):1518-1529.

16. Butcher LM, Beck S: AutoMeDIP-seq: a high-throughput, whole genome, DNA methylation assay. Methods 2010, 52:223-231.

17. Feinberg AP, Irizarry RA: Evolution in health and medicine Sackler colloquium: Stochastic epigenetic variation as a driving force of development, evolutionary adaptation, and disease. Proc Natl Acad Sci U S A 2010, 1(Suppl 107):1757-1764

18. El-Maarri O, Walier M, Behne F, van Uum J, Singer H, Diaz-Lacava A, Nusgen N, Niemann B, Watzka M, Reinsberg J, van der Ven H, Wienker T, StoffelWagner B, Schwaab R, Oldenburg J: Methylation at global LINE-1 repeats in human blood are affected by gender but not by age or natural hormone cycles. PLoS One 2011, 6:e16252

19. Choi SH, Worswick S, Byun HM, Shear T, Soussa JC, Wolff EM, Douer D, Garcia-Manero G, Liang G, Yang AS: Changes in DNA methylation of tandem DNA repeats are different from interspersed repeats in cancer. Int J Cancer 2009, 125:723-729.

20. Fryer AA, Emes RD, Ismail KM, Haworth KE, Mein C, Carroll WD, Farrell WE: Quantitative, high-resolution epigenetic profiling of $\mathrm{CpG}$ loci identifies associations with cord blood plasma homocysteine and birth weight in humans. Epigenetics 2011, 6:86-94.

21. Wong CC, Caspi A, Williams B, Craig IW, Houts R, Ambler A, Moffitt TE, Mill J: A longitudinal study of epigenetic variation in twins. Epigenetics 2010, 5:516-526.
22. Lam LL, Emberly E, Fraser HB, Neumann SM, Chen E, Miller GE, Kobor MS Factors underlying variable DNA methylation in a human community cohort. Proc Natl Acad Sci U S A 2012, 2(Suppl 109):17253-17260.

23. Schneider E, Pliushch G, El Hajj N, Galetzka D, Puhl A, Schorsch M, Frauenknecht K, Riepert T, Tresch A, Muller AM, Coerdt W, Zechner U, Haaf $\mathrm{T}$ : Spatial, temporal and interindividual epigenetic variation of functionally important DNA methylation patterns. Nucleic Acids Res 2010 38:3880-3890.

24. Bock C, Walter J, Paulsen M, Lengauer T: Inter-individual variation of DNA methylation and its implications for large-scale epigenome mapping. Nucleic Acids Res 2008, 36:e55.

25. MacDonald JR, Ziman R, Yuen RK, Feuk L, Scherer SW: The Database of Genomic Variants: a curated collection of structural variation in the human genome. Nucleic Acids Re 2014, 42:D986-D992.

26. Rozen S, Skaletsky H: Primer3 on the WWW for general users and for biologist programmers. Methods Mol Biol 2000, 132:365-386.

27. Mann HB, Whitney DR: On a test of whether one of two random variables is stochastically larger than the other. The annals of mathematical statistics 1947, 18(1):50-60.

28. Lance GN, Williams WT: A general theory of classificatory sorting strategies II. Clustering systems. The computer journal 1967, 10:271-277.

\section{doi:10.1186/s13039-014-0073-8}

Cite this article as: loannides et al:: Inter-individual methylation variability in differentially methylated regions between maternal whole blood and first trimester CVS. Molecular Cytogenetics 2014 7:73.

\section{Submit your next manuscript to BioMed Central and take full advantage of:}

- Convenient online submission

- Thorough peer review

- No space constraints or color figure charges

- Immediate publication on acceptance

- Inclusion in PubMed, CAS, Scopus and Google Scholar

- Research which is freely available for redistribution 